\title{
La transdiciplinariedad de la ética en las ingenierías
}

Transdisciplinarity of ethics in engineering

ollspyed, this f, 밀.

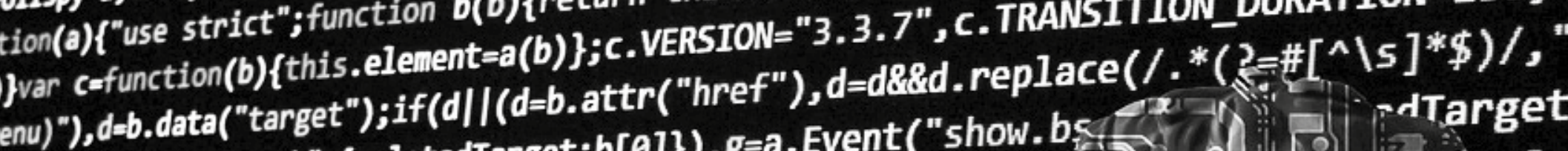
enu)"), d=b.data( target"); a.Event("hide.bs.tab", \{relatedTarget:b[0]\}), ented()) \{var h=a(d); this.activate(b.closest("1i"),c), this . are type: "shown.bs.tab", relatedTarget:e[0]\})\})\}\}\},c.prototype ve").renoveclass("active").end().find(' [data-toggle="tab'

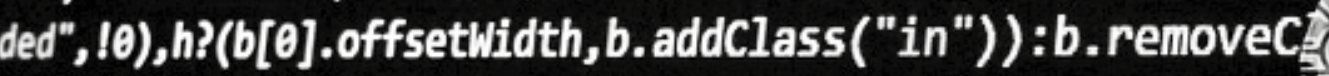

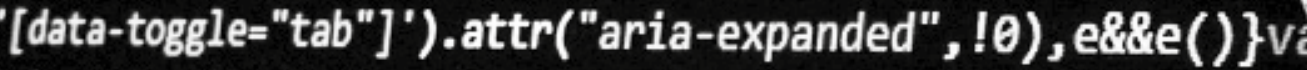
.find(") .fade").length);g.length\&\&h?g.one("bsTransition" fn.tab;a. fn. $t a b=b, a . f n . t a b$. Constructor=c, a. fn. tab. nocon: a(document).on("click.bs.tab.data-api", '[data-toggle="t? ;function b(b)\{return this.each(function() \{var $d=a$ (thi \&e[b]()\})\}var cafunction(b,d) $\{$ this.options=a.extend \{\} y(this.checkposition, this)).on("click.bs.affix.data-api" ? inction $(a, b, c, d)\{$ var e=this, starget ethis. affixed)return null starget. $\operatorname{scrol1Top}(), f=t h i s . \$$ elem top": null! =d\&8i+j>=a-d\&8"bott ("affix");var a=this.\$target, , prototype.getPinne ion() \{setTimeout (a. target. scrollTop(), b=this ions.offset, $e=d$. proxy(this. checkPos it

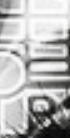




\title{
La transdiciplinariedad de la ética en las ingenierías ${ }^{1}$
}

\section{Transdisciplinarity of ethics in engineering}

\author{
Jorge Enrique Herrera Rubio², Karla Yohana Sánchez Mojica ${ }^{3}$ \\ Universidad de Pamplona - Fundación de Estudios Superiores Comfanorte FESC, Cúcuta, Colombia
}

Artículo recibido en agosto del 2016; artículo aceptado en noviembre de 2016

Citación del artículo: Herrera, J. \& Sánchez, K. (2017). La transdiciplinariedad de la ética en las ingenierías. I+D

Revista de Investigaciones, 9(1), 70 - 81

\begin{abstract}
Resumen
El presente artículo es el resultado de un estudio experimental realizado en una población base con el cual se pretende analizar la apreciación y la percepción que los miembros de dicha población tienen sobre la ética en los diferentes campos de las ingenierías. El estudio está fundamentado en la transdisciplinariedad de la ética la cual se aplica y se extiende a las diferentes actividades del ser humano, de acuerdo a las siguientes dimensiones: cultura científica, la tecnociencia, la cultura moderna, la globalización, el ciberespacio, la bioética y la ética, apoyado en la perspectiva de diferentes autores. Los resultados - producto de la aplicación de un instrumento de medición- se analizan a partir de un diseño metodológico de tipo exploratorio, para finalmente inferir sobre la impresión que los individuos tienen sobre la ética en las ingenierías.
\end{abstract}

Palabras clave: Cultura, moral, disciplina, ingeniería, ontológico.

\begin{abstract}
The present article is the result of an experimental study carried out in a base population which is tried to analyze the appreciation and the perception about the ethlics in the different fields of the engineering The study is based on the transdisciplinarity of ethics which is applied and extended to the different activities of the human being, according to the following dimensions: scientific culture, techno science, modern culture, globalization, cyberspace, Bioethics and ethics, it is supported by the perspective of different authors. The results - product of the application of a measurement instrument - are analyzed from a methodological design of an exploratory type, to finally infer about the impression that the individuals have on the ethics in the engineering.
\end{abstract}

Keywords: Culture, moral, discipline, engineering, ontological.

1. Artículo de investigación, el estudio realizado es catalogado como experimental y exploratorio, resultado de un proyecto de investigación, perteneciente al área de Ingenierías y arquitectura, subárea de Ingeniería, desarrollado por los grupos de investigación Gitent y Grinfesc, fue financiado por la Universidad de Pamplona y la Fundación de Estudios Superiores Comfanorte FESC de la ciudad de Cúcuta (Colombia). Dirección: Pamplona, Norte de Santander y Av. 4 n. ${ }^{\circ}$ 15-14 Playa (respectivamente). Fecha de inicio: 29 de febrero de 2016, fecha de finalización: 2 de agosto de 2016.

2. Doctor en Ciencias de la Universidad Rafael Belloso Chacín, magister en Electrónica de la Universidad Experimental del Táchira, docenteinvestigador del grupo de investigación Gitent Facultad de Arquitectura elngenierías de la Universidad de Pamplona, Pamplona - Norte de Santander (Colombia): PBX: 5685303. Correo institucional: jherrera@unipamplona.edu.co.

3. Ingeniera en Telecomunicaciones de la Universidad de Pamplona, especialista en Práctica Pedagógica Universitaria de la Universidad Francisco de Paula Santander, directora de investigaciones-investigadora del grupo de investigación Grinfesc de la Fundación de Estudios Superiores Comfanorte FESC, Cúcuta (Colombia): Av. 4 n. 15-14 Playa, PBX: 5829292, ext. 228. Correo institucional: investigaciones@fesc.edu.co 
El ejercicio práctico de la ingeniería en las organizaciones se refleja en los procesos productivos que se establecen en los planes estratégicos, por medio del desarrollo de las competencias de tipo gerencial y de liderazgo con el fin de orientar la misión y la visión de las organizaciones, resaltando los valores éticos tales como la honestidad, la responsabilidad, la justicia, la equidad, entre otros; en el desempeño de sus funciones.

Es importante hacer una reflexión sobre las competencias profesionales de los futuros ingenieros para saber cómo las exigencias y las necesidades actuales de la sociedad les permiten desenvolverse, para trabajar en forma razonada en entornos cambiantes y de incertidumbre. Desde el punto de vista del liderazgo ingenieril empresarial se asumen responsabilidades de manera voluntaria para ejercer la autoridad y guiar a otros individuos en la toma de decisiones, muchas veces de forma desacertada, sin tener en cuenta los intereses de la organización con el fin ayudar en el cambio organizacional; es allí en donde la cuestión ética va más allá de la competencia profesional y se traslada al escenario del comportamiento y el actuar humano.

Por tal razón, se analizan algunos aportes de autores sobre el tema de la ética; desde el punto de vista de los principios y valores para la Academia Española (2015) se puntualiza que la ética se puede interpretar como: "1. conjunto de normas morales que rigen la conducta de la persona en cualquier ámbito de la vida. Ética profesional, cívica, deportiva o es la 2) parte de la filosofía que trata del bien y del fundamento de sus valores". Al respecto, Luer (2014) afirma: "para influir en otros es prioridad comenzar a cambiar la forma de actuar por uno mismo. Una vez que cultivamos y reconocemos a nuestro líder interior, podemos comenzar la proyección hacia afuera de ese líder e influir en los demás de manera positiva". En esta manera, se observa que existe una reflexión sobre los valores y principios en el interior de cada individuo.

Considerando el estudio de la ética desde un contexto holístico que compromete el análisis del ser humano y las organizaciones en el ambiente en que se desarrollan, podemos analizar como referente la comprensión de la bioética, tal como lo expresa Maldonado (2001), quien describe la Bioética como un estudio interdisciplinario de los procesos investigativos, de los avances técnico científicos y de su aplicación en el mediano y largo plazo con la pretensión de valorar éticamente su impacto positivo de las investigaciones científicas sobre el desarrollo y la estructura misma de la vida. Lo anterior supone que no solo es posible entender y apropiarse del conocimiento, sino que también se debe comprender la esencia de la naturaleza del ser humano en su conjunto.
Por otra parte, el proceso acelerado que está viviendo la humanidad producto de las investigaciones para dar solución a problemas generacionales, hace que se susciten nuevas formas de reflexión sobre asuntos complejos, al respecto Morin (2011, p. 63) describe que "podemos decir que aquello que es complejo recupera, al mundo empírico, la incertidumbre, la incapacidad de lograr la certeza, de formular una ley, de concebir un orden absoluto", es decir, todo aquello que es considerado racional en una situación compleja, puede generar posturas contradictorias que producen nuevas formas de análisis y reflexión profunda de la realidad.

Las disciplinas tecnológicas que se ayudan de las ciencias experimentales como es el caso de la física y la química, dan solución a problemas o fenómenos como procesos independientes para la construcción de nuevos conocimientos, de manera que cada disciplina en particular busca una especialización propia (monodisciplina), pero, no se puede desconocer que también se hace extensivo el apoyo de otras disciplinas que por la necesidad epistemológica necesitan de un sustento en conjunto; en torno al tema Morin (citado por Bedate, 2014, p. 219), recomienda que la "interdisciplinariedad puede significar intercambio y cooperación" como una integración de la producción de conocimiento, y es también notorio en las disciplinas científicas la diversificación como complemento de cooperación para generar compatibilidad entre ellas.

En el avance de las ciencias se busca un conocimiento lo más completo posible que permita la integración entre las diferentes disciplinas científicas ante un problema complejo, situación que se puede considerar desde el punto de vista transdiciplinar para lograr un conocimiento complementario, que según Morin (1996) se basa en los tres pilares de la transdisciplinariedad: los niveles de realidad, la lógica del tercero incluido y la complejidad, que determinan la metodología de la investigación transdisciplinaria.

Con base en lo anterior, según Morin la investigación transdisciplinaria que corresponde a cierto grado de transdisciplinariedad se acercará más bien a la multidisciplinariedad (como en el caso de la ética); la de otro grado -a la interdisciplinariedad (como en el caso de la epistemología); y la de otro grado más -a la disciplinariedad (1996, p. 38).

En la Figura 1 se observa cómo funciona el proceso de la interdependencia del conocimiento para las diferentes disciplinas y los tres pilares de la transdisciplinariedad. 


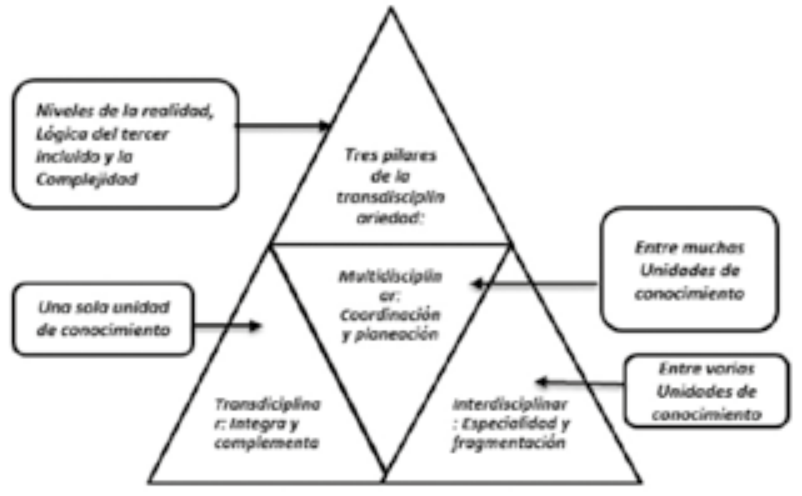

Figura 1.

Esquema de un proceso de la transdisciplinariedad. En el estudio, cómo funciona el proceso de la interdependencia del conocimiento para las diferentes disciplinas, por autores de la investigación.

Si extrapolamos el concepto la disciplinariedad en la integración de la ética en las ingenierías, se puede ver que existe una conexidad integral de los diferentes tipos conocimiento de ética para dar respuestas a interrogantes de problemas complejos.

Vinculado al concepto, Pérez, R. (2010, p. 129) define la ética como "la disciplina que trata de lo que es bueno y malo, del deber, la responsabilidad y la obligación moral. La ética también puede ser vista como un grupo de principios y valores morales". La moralidad es una doctrina o sistema de conducta moral. La conducta moral se refiere a aquello que se relaciona a principios de lo correcto y lo incorrecto en la conducta. Llanos (2014), por su parte, sostiene que la ética es la forma de permitir que los actos de una persona se basen en principios y valores, lo que redunda en beneficio propio y en la sociedad; es de anotar que, la conducta fundamental en todas las actividades humanas promueven los comportamientos moralmente correctos que deben tener los individuos entre sí, y es lo que se denominan los códigos deontológicos.

\section{Contextos éticos}

El diseño de un bosquejo general sobre la ética se sintetiza según los siguientes contextos:

\section{Ética en la tecnociencia}

De acuerdo con los análisis de Exteberria (2008, p. 13) existe un ambiguo protagonismo de la ética que sigue a una cierta marginación sufrida en el pasado reciente: Hasta hace relativamente poco tiempo, parecía que la tecnociencia amenazaba con sustituir a la ética. La primera se nos presentaba, en efecto, como un conocimiento fiable y fecundo, como saber y transformación de lo que se sabe, para resolver nuestros males físicos y sociales.

Esto no significa que la tecnociencia pueda llegar a solucionar problemas de los individuos, mientras no se considere la dignidad humana como eje central de los problemas de la sociedad.

Díaz (2007) también enumera algunos aspectos de mayor impacto de la tecno ciencia: 1) en la economía (como son: las exigencias de las reglas del mercado, la pronta entrega de productos y la obsolescencia de sus productos y servicios) y 2) en la sociedad (el devenir de la política, procesos de industrialización se globalizan, la explotación de recursos naturales y la maquinaria bélica). Dichos aspectos afectan el proceder axiológico de la sociedad que implica deterioro de los valores, morales, éticos y espirituales.

\section{Ética en la cultura moderna}

De acuerdo con Ponce (2011), quien se refiere al concepto de cultura moderna citando a Habermas, quien la define como un periodo de tiempo que se define por la novedad en donde se rompen los esquemas del modo continuo histórico de cómo suceden las cosas, de todo aquello que es tradicional, logrando conciencia de lo que se debe ser, mediante la adecuación de cambios presentes ante las nuevas necesidades, sin considerar los preceptos establecidos con el fin de olvidar los valores éticos y morales.

Con referencia a la cultura moderna, para Exteberria (2008) es conveniente resaltar tres rasgos relacionados con la ética: 1) el de la secularización, como fenómeno complejo que tiene en cuenta solo la pérdida de la referencia religiosa para la organización de la convivencia; 2) el de la autonomía, cuando la religión ha dejado de ser referencia pública para la convivencia ciudadana y se ha pasado a ser referencia opcional de sentido para la realización de los ideales de vida; 3 ) el del pluralismo, el tercer rasgo de la modernidad deriva evidentemente del segundo.

\section{Ética en la globalización}

El efecto de la transculturización, los conflictos globales, loscomportamientos del mercado, la internacionalización de la economía, la interdependencia económica y política entre las naciones como consecuencia de tratados internacionales, han permeado el "sistema de valores" redefiniendo los nuevos espacios de convivencia social; al respecto, García (2011) afirma que la ética es y debe ser 
parte constitutiva de todo el proceso de transformación económica que caracteriza al actual "episodio históricocultural de la globalización actual". No se puede separar la lógica económica de la lógica ética porque interactúan simultáneamente.

En un contexto más general Morin (2011) interpreta el fenómeno de la globalización como un carácter antiético en el concepto. ¿Por qué? Porque en las sociedades llamadas "desarrolladas" podemos ver la desintegración de las solidaridades tradicionales de la gran familia, del barrio, de las comunidades y la desaparición de las solidaridades concretas por el afán desmedido por un desarrollo de mercado por encima de una cultura.

En síntesis, Exteberria (2008, p. 19) expone dos dimensiones relevantes de la ética en la globalización:

1) La primera de ellas nos pone de nuevo en contacto con el reto de la justicia: el mundo se globaliza generando un fuerte porcentaje de excluidos, con el impacto que se precise en las estructuras económicas y política; 2 ) la segunda de ellas nos acerca al pluralismo, pero con otros matices, como pluralismo cultural.

Lo anterior, puede generar dos connotaciones especiales: 1) la valoración de la ética de un modo más general porque entran en juego dentro los derechos humanos fundamentales de los individuos y 2) la división de las expresiones culturales propias de cada nación al imponerse un modelo de pluralismo cultural.

\section{Ética en el ciberespacio}

Es innegable que la Internet, como la red de redes, ha influido técnica y tecnológicamente en el desarrollo y evolución de la humanidad en todos los campos, hasta el punto se ha llegado a pensar que su ilimitado uso ha trasgredido los espacios de la intimidad individual y hasta la de una nación.

De acuerdo con el educador y periodista Estremadoyro (2012), en el mundo de la Internet o ciberespacio uno de los dilemas es cómo saber si una versión de la información es real o es probablemente un engaño. Es decir, la credibilidad del material expuesto en el ciberespacio genera desconfianza y desinformación, por tanto, se debe recurrir a las fuentes originales y reales de quien cita y respalda la información.

La percepción del mundo real se pierde cuando no existe un uso responsable de la información y la materialización de los actos de los individuos; en este escenario del ciberespacio, según Pérez (2008, p. 27):
(...) estas consideraciones implican que la técnica anula incluso a la propia ética e impide que el sujeto humano se pregunte por su propia identidad. Provoca no sólo las descalificaciones propias de quienes consideran eternos los valores éticos, sino que se establece un estado de desfiguración de la ética.

Por tal razón, se debe evaluar el tipo de información, descubrir razonablemente los temas que se investigan y a las personas que las publican, hasta llegar a las fuentes primarias de la verdad para poder discernir axiológicamente un determinado asunto.

\section{La bioética como ética aplicada en la ciencia}

Según la Real Academia Española (2015), la bioética se basa en los estudios de los problemas éticos originados por la investigación biológica y su aplicación en la ingeniería genética; por su parte, para a García (2015) la bioética es la ética aplicada a la sociedad del siglo XXI y su origen se halla en dos corrientes: 1) una surgida en Wisconsin, con Potter, quien establece una visión holística al concebirla como la ciencia de la supervivencia de todas las especies existentes. 2) La otra corriente nace en Georgetown, con Helleger, quien considera una ética médica aplicada que trata de resolver los problemas que se presentan en la práctica médica cotidiana por medio de la ingeniería genética.

Por su parte, Dolores (2009) identificar y analizar los aspectos éticos implicados en la relación clínica y distingue los cuatro principios básicos de la Bioética, para ello destaca los aportes de Beauchamp y Childress, dos grandes autores en Bioética reconocidos fundamentalmente por establecer los cuatro grandes principios, que a su juicio son asumibles por todos los seres racionales: la no-maleficencia, la beneficencia, la autonomía y la justicia. Sin embargo, para Beauchamp estos cuatro principios son moralmente exigibles porque sus consecuencias son buenas, mientras que para Childress estos principios son realmente objetivos y por tanto deben exigirse siempre independientemente de sus consecuencias.

Con el fin de aclarar los principios fundamentales de la bioética, en la Figura 2 se detalla la metodología. 


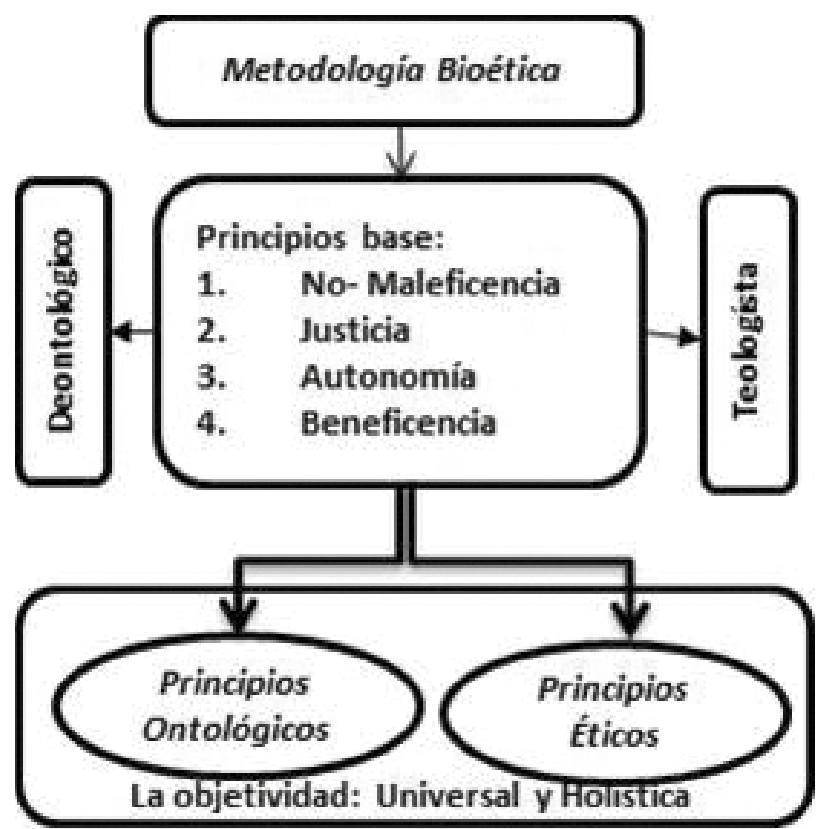

Figura 2.

Esquema de los principios de la metodología bioética. En el estudio, se detalla la metodología que se fundamente en los principios fundamentales.

Fuente: Elaboración propia

\section{Los principios de la Bioética}

La metodología bioética es un sistema de referencia de carácter formal expresado en dos principios: el principio ontológico, según el cual "el ser humano es persona y en cuanto tal tiene dignidad y no precio"; y el principio ético, el cual sostiene que "en cuanto que personas, todos los seres humanos son iguales y merecen la misma consideración y respeto". Este sistema de referencia se propone como "objetivo", es decir como exigible siempre, universalmente: estos dos principios son válidos para cualquier ser humano y en toda situación, o lo que es lo mismo, no tienen excepciones. Todo lo anterior es un intento de interpretación para mediar entre las prácticas más arraigadas: el deontologismo y el teologismo, para llegar a una objetividad universal y holística.

Los cuatro principios enunciados por Beauchamp y Childress son actualmente aceptados en el discurso de la Bioética y quedan expresados de la siguiente manera:

- No maleficencia, que obliga a no dañar a los demás -primum non nocere- y por lo tanto, a realizar correctamente el trabajo profesional.

- Justicia, que obliga a proporcionar a todos las mismas oportunidades en el orden de lo social; no discriminando, segregando o marginando.

- Autonomía, que exige reconocer que todas las personas, mientras no se demuestre lo contrario, son capaces de tomar decisiones y disponer de sí mismas libremente.

- Beneficencia, que exige hacer el bien, según los "criterios de bien" del posible beneficiado o paciente y a representarle cuando éste no pueda hacerlo por sí mismo.

\section{La ética aplicada o ética profesional en ingeniería}

La ética aplicada es una reflexión de los diferentes tipos o productos morales que surgen de las necesidades prácticas del actuar de una profesión para proponer soluciones basadas en la razón de una forma responsable. Al respecto, Tapia (2013) afirma que para lograr una mayor especialización del campo de acción o profesión se utilizan los códigos de ética, los cuales no son más que una compilación de las normas y reglas que determinan el comportamiento ideal o más apropiado para un grupo específico de profesionales.

En cambio, para Palazzi y Román $(2015$, p. 76) la ética aplicada es interdisciplinar, "por ello hoy en día es fundamental institucionalizar co-regulaciones morales, jurídicas y políticas, para que la ética no sea tan ineficiente; para que la política vaya más allá y vea más allá, para que la ética pueda incidir más acá". De acuerdo a lo anterior, se debe considerar una relación de todas las estructuras de las actividades humanas, atendiendo los cambios de los valores, reglas y principios que sean sostenibles ante la complejidad de las situaciones que se puedan adaptar a lo circunstancial y eventual.

Igualmente, para López (1997) la ética aplicada considera que los principios equivalen a mandatos óptimos, es decir, aquellos que prescriben algo que pueden realizarse en mayor o menor grado; en cambio las reglas corresponden a mandatos definitivos, funcionan como todo o nada, existiendo una relación más abierta en los principios y más cerrada para las reglas.

En Colombia, por medio de la Ley 842 de 2003, se reglamentó el ejercicio de la ingeniería y profesiones afines, y se adoptó el código de ética profesional con el fin de que el profesional de la ingeniería actué de forma ética; sin embargo, la ingeniería como una profesión, según Díaz (citado en Valencia, 2015) a diferencia de otras disciplinas (como la física o la filosofía) no es una unidad discursiva discreta y especializada con su propio campo intelectual, en tanto que la ingeniería busca su aplicación fuera de sí misma. De ahí que la ausencia de ética en el ingeniero afecta la convivencia con las personas que conforma el entorno: familia, empresa y sociedad. 


\section{Método}

\section{Tipo de estudio}

El estudio realizado es catalogado como empírico y exploratorio, pues busca conocer la realidad a partir de una información primaria acerca de lo que significa la ética. El proceso se inicia con la realización de un cuestionario aplicado a cada uno de los participantes, para evaluar el actuar ético de los futuros profesionales $y$ de los profesionales que ejercen sus roles en cada una de sus disciplinas; la idea general es la de analizar los resultados de la exploración producto de los imaginarios de la ética de cada uno de los participantes.

En la búsqueda de acerarse a la ética como un proceso de investigación exploratorio, Barrera (2015) explica que se requiere de un exhaustivo y laborioso proceso de recolección de datos en campo, donde se apliquen instrumentos inestructurados a variedad de fuentes para recolectar la mayor cantidad de información posible con respecto a los diversos eventos.

El uso de una metodología basada en las experiencias reales de los individuos objeto del estudio, según Cowton (citado por Rodríguez, 2006) proporciona un entendimiento del mundo tal como es y brinda una contribución esencial al análisis ético normativo. Esto es beneficioso no solo en la conducción del análisis, sino también en la comunicación, por el uso de ejemplos reales o realistas. La investigación propuesta permite hacer los análisis, las discusiones y las generalizaciones, aplicando métodos empíricos.

Para lograr el objetivo se elaboró una encuesta tomando como premisa la investigación documental inicial; el tema se segmentó en cinco tipos de comportamientos éticos: lo cívico, la tecno ciencia, la cultura moderna, la globalización y el ciberespacio. Las preguntas fueron codificadas con valoraciones cualitativas, después fueron recodificadas (cuantitativamente) para ser tabuladas en una hoja electrónica $y$, finalmente, se analizó la composición de la escala y los resultados por grupo de preguntas de acuerdo al grado de impacto esperado según las dimensiones éticas.

\section{Participantes}

Se empleó un instrumento tipo encuesta mediante el consentimiento informado, sobre una población aproximada de 1172 estudiantes de ingenierías en las áreas de alimentos, eléctrica, mecatrónica, telecomunicaciones, industrialy sistemas, el cual se realizó en forma aleatoria a 249 estudiantes (el equivalente al
$21,2 \%$ aproximado de la población analizada), el error de la muestra fue del 6,024\%, (6 encuestas incompletas y 9 encuestas erradas.

\section{Instrumentos}

Para el proceso analítico se elaboró un cuestionario de quince preguntas validadas por un grupo de cuatro expertos; las preguntas se codificaron inicialmente en forma cualitativa, luego se agruparon por componentes éticos y finalmente por dimensiones éticas. El orden establecido fue: totalmente en desacuerdo con un valor de 1; en desacuerdo, con valor de 2; neutral, con un valor de 3; de acuerdo, con un valor de 4, y totalmente en desacuerdo, con un valor de 5 . El cuestionario se estructuró en cinco componentes éticos y se determinaron tres tipos de dimensiones éticas respectivamente, como se observa en la Tabla 1.

\section{Resultados}

\section{Efecto de la ética de lo cívico}

Para la pregunta 1, un porcentaje del $57 \%$ de la población estudiantil es consciente del efecto negativo de plagiar en los exámenes con fin de evadir una responsabilidad como estudiante; por otra parte, cuando se pregunta si mentir es una ventaja para pasar por encima de cualquier circunstancia (pregunta 2), se observa que hay una actitud de neutralidad o conformismo en el 29\%; pero un $33 \%$ está totalmente en desacuerdo con esta situación y un $31 \%$ está en desacuerdo en mentir; finalmente, para (la pregunta 3) se advierte que el 35\% de los encuestados tienen claros los principios y valores éticos (están de acuerdo), el 30\% está totalmente de acuerdo en tener claridad con los principios y valores y el $23 \%$ se siente indiferente sobre dicho asunto. Según los resultados obtenidos, en las tres dimensiones del componente ético Cívico, hace falta fortalecer más el criterio personal en lo que respecta a transparencia, honestidad y justicia, como se observa en la Figura 3.

\section{Efecto de la ética de la tecnociencia}

En la Figura 4 se aprecia que un $36 \%$ de los encuestados consideran la tecnología como parte de la solución de los problemas sociales (pregunta 4) y un $40 \%$ es razonable ante dicha pregunta; ahora, frente a la situación en que la tecnología es una amenaza cuando no se limita (pregunta 5), un $41 \%$ se considera imparcial, al parecer no tienen en cuenta los riesgos que la tecnología puede ocasionar al no manejar de forma responsable los recursos tecnológicos en beneficio de la sociedad y un $31 \%$ ve como una amenaza el uso desmedido de 
Tabla 1

Tabulación de los resultados de las dimensiones éticas y sus componentes

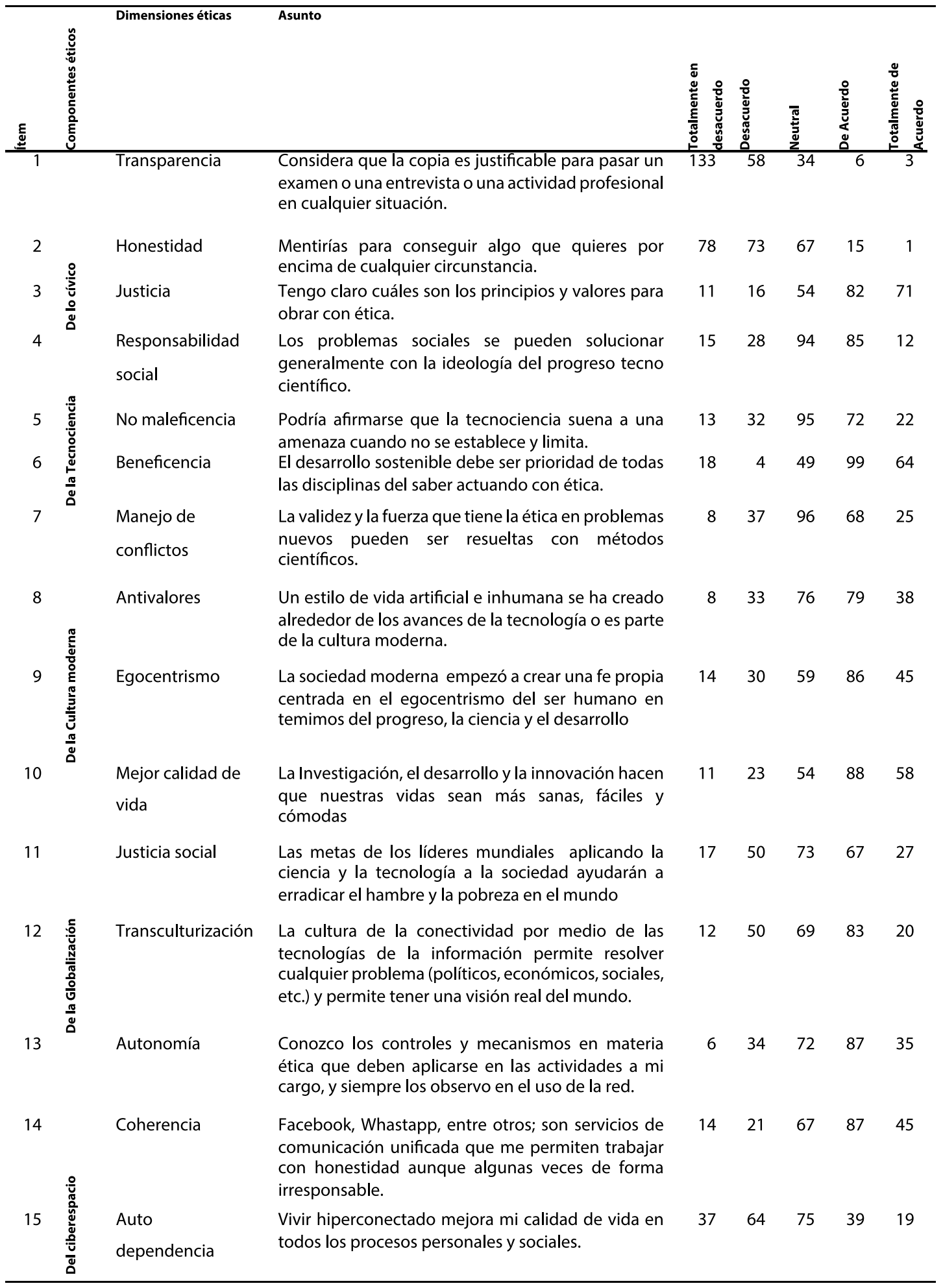


la tecnología; en la (pregunta 6) el desarrollo sostenible producto de la tecnociencia es importante para el $42 \%$ (está de acuerdo) de la población si se actúa con ética, el $27 \%$ está totalmente de acuerdo y un $21 \%$ le es indiferente. Para los encuestados el componente ético la tecnociencia puede ser valiosa cuando se obra con responsabilidad social y se respetan la pluralidad de los valores en beneficio de la sociedad.

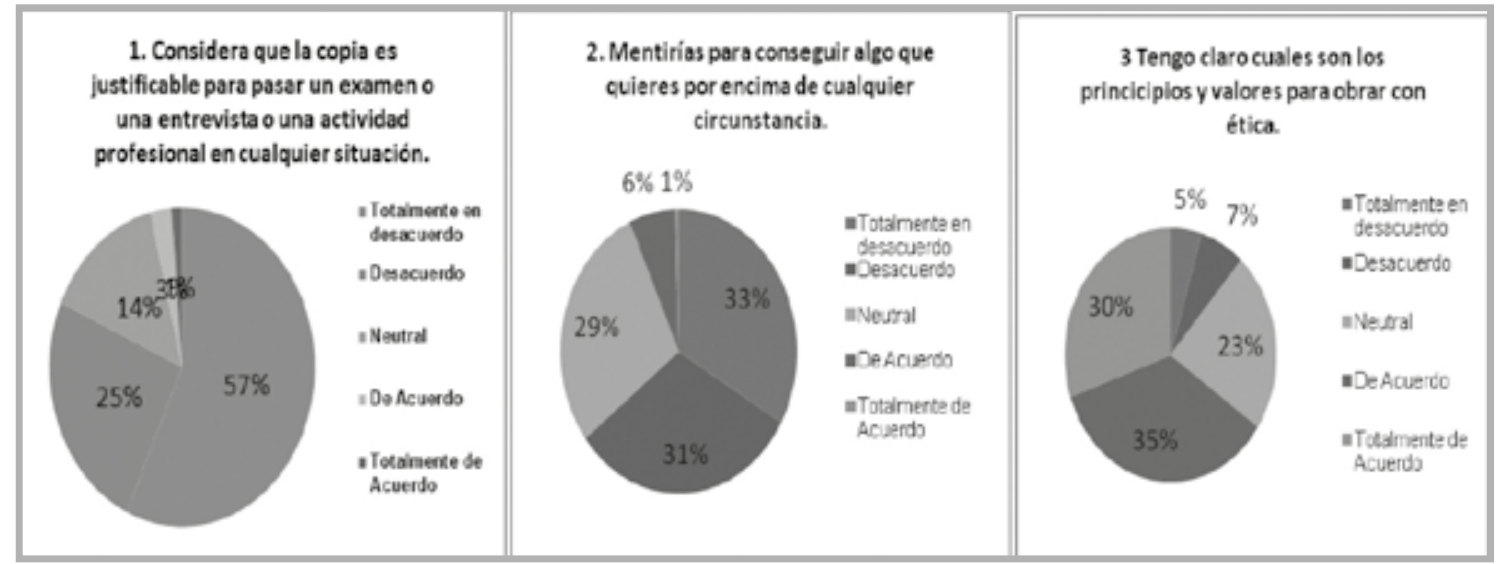

Figura 3.

Resultados del componente ético Cívico. Aplicación del instrumento de recolección de información fase 1 del estudio de investigación, por autores de la investigación.

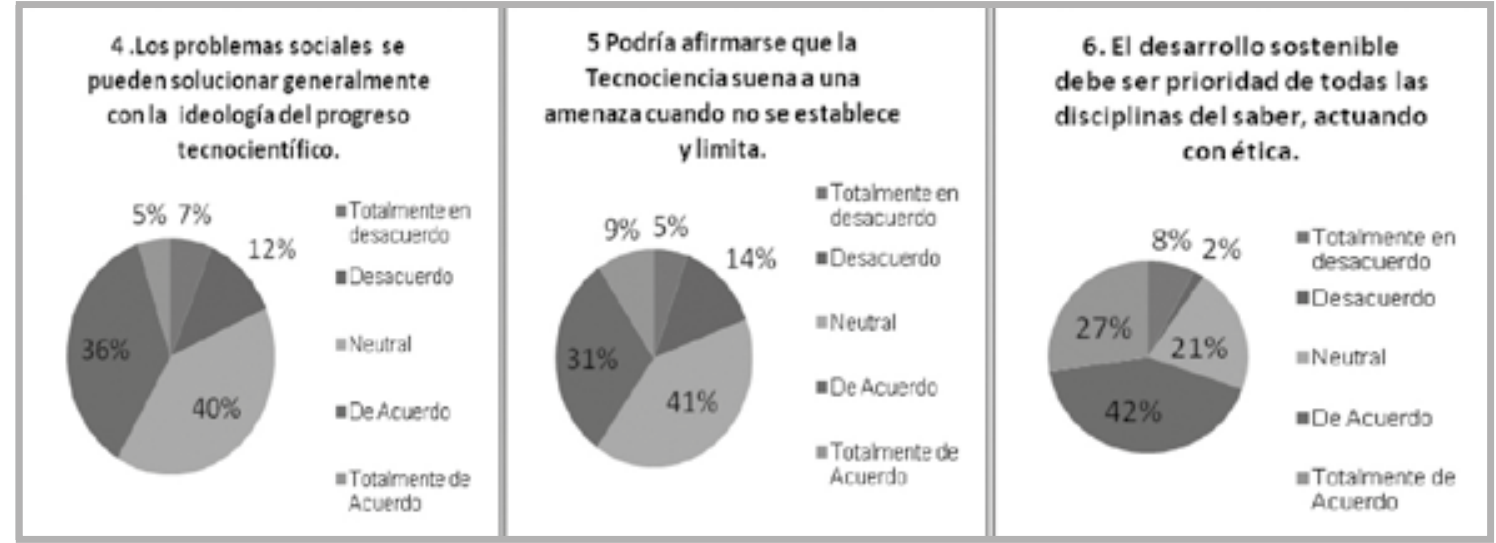

\section{Figura 4.}

Resultados del componente ético de la Tecnociencia. Aplicación del instrumento de recolección de información fase 1 del estudio de investigación, por autores de la investigación.

\section{Efecto de la ética de la cultura moderna}

En el caso de los componentes de la cultura moderna (pregunta 7), la validez y la fuerza que tiene la ética en la solución de problemas con métodos científicos; el $41 \%$ advierte una imparcialidad al dejar en manos de la ciencia la solución a todos los problemas. Solo el $29 \%$ considera estar de acuerdo en que la ciencia puede ayudar en el desarrollo y el progreso humano, el $16 \%$ se siente en desacuerdo y solo un $11 \%$ está totalmente de acuerdo.

Con referencia a la pregunta 8 , respecto al estilo de vida artificial e inhumano creado alrededor de los avances tecnológicos, el $34 \%$ de los encuestados están de acuerdo, al $33 \%$ le parece indiferente, un 14\% está en desacuerdo y un $16 \%$ está totalmente desacuerdo; al interrogante de la pregunta 9 , sobre la fe centrada en el egocentrismo en el ser humano solo el $37 \%$ está de acuerdo, un $25 \%$ tiene una postura de indiferencia y un $19 \%$ dice estar totalmente de acuerdo, es decir, se percibe una falta de autoconciencia, ambiciones desmedidas y una distorsión de la realidad; ante estos interrogantes el componente ético de la cultura moderna muestra un conflicto de intereses y de antivalores frente a las reglas sociales, existe un desconocimiento de las normas establecidas socialmente como positivas para el bien común, predominan los intereses particulares, 
se deteriora la confianza y el respecto por parte del ser humano hacia los demás, como se aprecia en la Figura 5.

\section{Efecto de la ética de la globalización}

Con base en la pregunta 10 de cómo la investigación, el desarrollo y la innovación (I+D+i) aportan beneficios al ser humano, solo el $25 \%$ se considera totalmente de acuerdo, el $37 \%$ dice estar de acuerdo, el $10 \%$ está en desacuerdo y el $23 \%$ es escéptico de cómo la $1+D+i$ produce beneficios a la sociedad; para la pregunta 11 al parecer existe una disposición de neutralidad del $31 \%$ con respecto a cómo la tecnología puede mejorar los problemas sociales, el $29 \%$ está de acuerdo con mejorar los problemas sociales, el $21 \%$ está en desacuerdo y solo el $12 \%$ totalmente de acuerdo; caso contrario sucede con la pregunta 12, de cómo la cultura de la conectividad por medio de las Tecnologías de la Información y la Comunicación (TIC) resuelven los problemas sociales, el $35 \%$ está de acuerdo por el uso que se le da a la tecnología, el 30\% tiene una sensación de imparcialidad o indiferencia, el $21 \%$ está en desacuerdo y solo el 9\% está totalmente de acuerdo con la cultura de la conectividad para resolver los problemas como se muestra en la Figura 6.

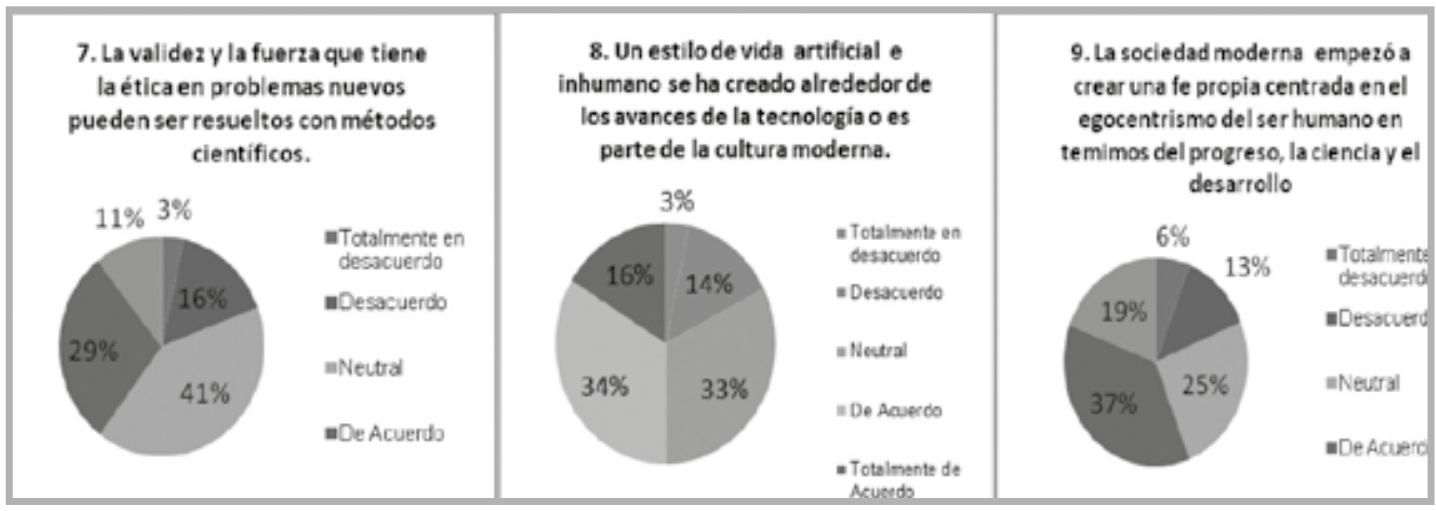

Figura 5.

Resultados del componente ético cultura moderna. Aplicación del instrumento de recolección de información fase 1 del estudio de investigación, por autores de la investigación.

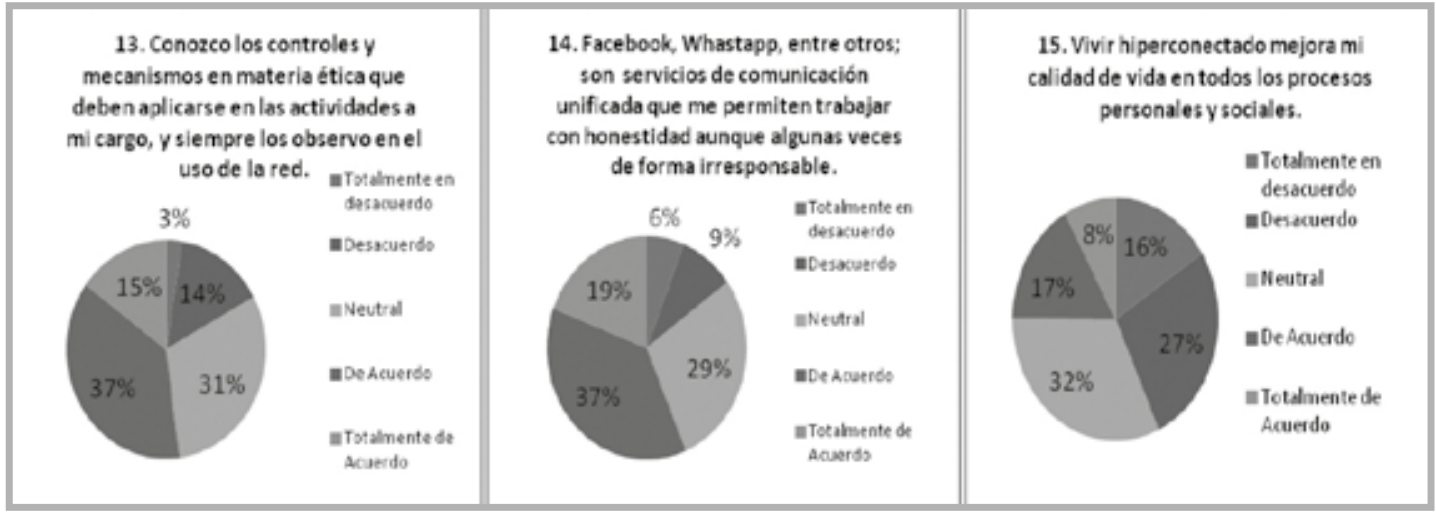

Figura 6.

Resultados del componente ético Globalización. Aplicación del instrumento de recolección de información fase 1 del estudio de investigación, por autores de la investigación.

\section{Efecto de la ética del ciberespacio}

Finalmente, para este componente ético, con respecto al conocimiento de controles y mecanismos en materia ética en las actividades laborales (pregunta 13) solo el $37 \%$ está de acuerdo en que se deben conocer los mecanismos, normas y reglas en las actividades profesionales que realiza el ingeniero ante el uso de la tecnología, el $37 \%$ lo considera normal, el $15 \%$ está totalmente de acuerdo y el $14 \%$ está en desacuerdo.

Los resultados de la pregunta 14 indican que el $37 \%$ de los encuestados está de acuerdo con que las redes sociales constituyen un apoyo para las actividades de tipo 
ingenieril, un $29 \%$ se siente neutral ante dicha respuesta, el $19 \%$ si está totalmente de acuerdo con el uso de las redes para dichos propósitos, el $9 \%$ está en desacuerdo y el $6 \%$ se considera totalmente en desacuerdo.

Ante la pregunta 15 , sobre si la hiperconectividad mejora la calidad de vida, solo el $32 \%$ está totalmente de acuerdo en que se mejoran las relaciones personales y sociales con el uso de la tecnología, un $27 \%$ está en desacuerdo, el $17 \%$ dice estar de acuerdo y sólo el 16\% está totalmente en desacuerdo, postura que permite entender que las dimensiones éticas de autonomía, coherencia y auto dependencia son situaciones muy personales, que posibilitan la necesidad de convivencia con los demás; pero en su conjunto el componente ético del ciberespacio, puede mejorar las relaciones interpersonales sin descuidar el crecimiento ético en los proceso personales y sociales, como se detalla en la Figura 7.

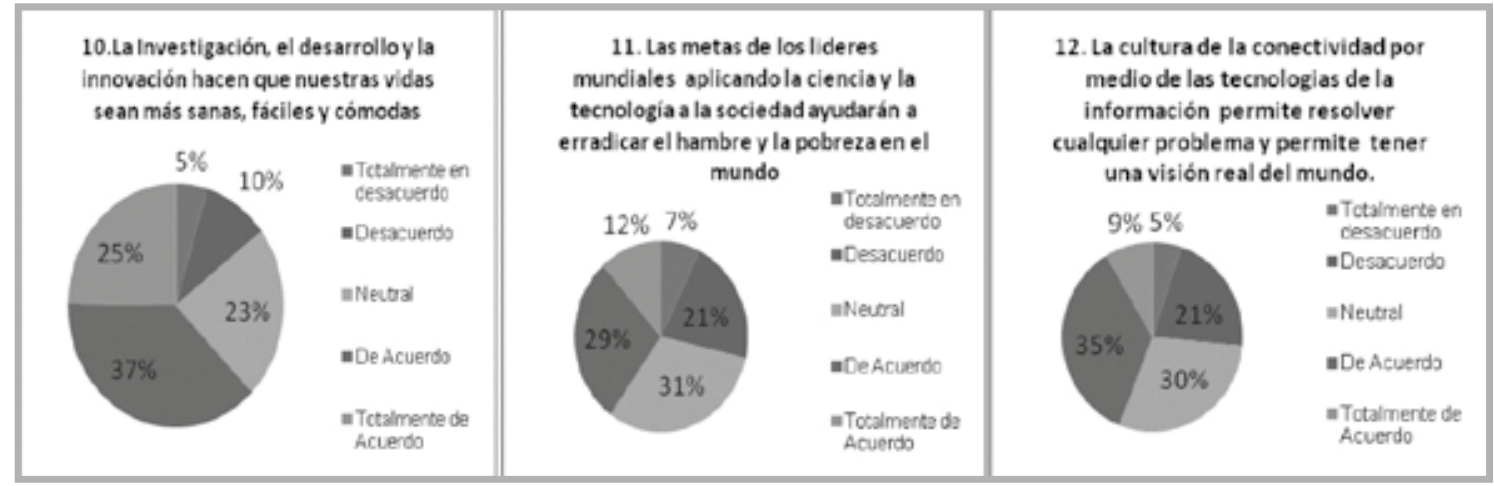

Figura 7.

Resultados del componente ético Ciberespacio. Aplicación del instrumento de recolección de información fase 1 del estudio de investigación, por autores de la investigación.

\section{Conclusiones}

La diversidad de disciplinas científicas se complementan por medio de una relación dinámica, que permite entender la interdependencia del conocimiento para las diferentes disciplinas, de ahí la importancia del papel transformador que trae consigo el nuevo conocimiento y que puede amenazar el valor de la ética en las diferentes actividades de las ramas de la ingeniería, cuando se busca el desarrollo científico en función de la humanidad y no se miden las consecuencias ante la sociedad.

La ética es un proceso interno que se reglamenta y se construye en cada persona de acuerdo sus principios y valores, y que se adquiere a lo largo del desarrollo tanto físico como intelectual, con el transcurso los años, para afrontar situaciones ante la realidad mediante una transformación personal de forma continua, de autocontrol y de autoevaluación permanente. De esta manera, se puede decir que la ética es una manifestación de conciencia interna y al mismo tiempo una manifestación reflexiva sobre los actos y experiencias, que se tienen de cómo actuar bien ante los demás.

La conveniencia de actuar en forma ética en el ejercicio de las ingenierías se refleja en los componentes éticos resultado de la investigación, para los cuales se requiere fortalecer: la honestidad, valorar la transparencia, respetar la pluralidad de los valores éticos, actuar con responsabilidad social, robustecer la confianza y respetar las normas morales. Todas las anteriores cualidades deben estar reflejadas en el ejercicio de la profesión y que de ninguna manera deben separarse de la rigurosidad que exige la ética ante las actividades diarias que se realizan.

Para la cimentación del proceso de una ética holística, debe conformarse un sistema mixto de responsabilidades entre las diferentes disciplinas científicas del saber por intermedio del canje de razones propositivas en la búsqueda de lograr la objetividad ética y moral de forma integral. Debe iniciarse un proceso axiológico del proceder y actuar de las generaciones futuras de acuerdo a la percepción que tienen del mundo y reestructurar las condiciones de convivencia en función de una sociedad más justa y equilibrada con un enfoque de bienestar global.

\section{Referencias}

Barrera, J. (2015). El proyecto de investigación. Comprensión holística de la metodología y la investigación. 
Bedate, A. (2014). El saber interdisciplinar (pp.216-223). Madrid: Universidad Pontificia.

Congreso de Colombia. (octubre, 9 de 2003). Ley 842 de 2003.

Díaz, E. (2007). Entre la tecnociencia y el deseo. La construcción de una epistemología ampliada (pp.130-159). Buenos Aires: Biblos.

Dolores, D. \& Marijuán. (2009). Curso: Bioética. OCW. Departamento de Especialidades Médico Quirúrgicas. Área Medicina Legal y Forense. Universidad del País Vasco. Recuperado 14 de marzo de 2016, de http://cvb.ehu.es/ open_course_ware/castellano/salud/bioetica/content/ ud2_metodo_ocw_09.pdf

Estremadoyro, J. (2012). Ética en el Ciberespacio. Revista Comunife, 12, 55-72. Perú. Recuperado 29 de julio de 2015, de http://www.unife.edu.pe/publicaciones/revistas/ compunicacion/comunife12/Julio\%20Estramadoyro.pdf

Etxeberria, X. (2008). Ética de las profesiones (pp.13-20). Sevilla: Universidad Jesuita.

García, B. (2015). La bioética como ética aplicada a la sociedad civil del siglo XXI. Recuperado 28 de agosto de 2015, de http://www.bioetica.org.ec/articulos/ articulo_la_ bioetica_como_etica_aplicada.htm.

García, S. (2011). Ética y globalización: su integración e institucionalización (problemas éticos en torno a la globalización).

López, M. (1997). Principios morales de la ética aplicada. Agora, 16(2), 159-160.

Luer, C. (2014). Cómo ejercer tu liderazgo para influenciar a los demás. Recuperado 14 de agosto de 2015, de http://www. merca20.com/como-ejercer-tu-liderazgo-para-influenciara-los-demas/.

Llanos, L. (2014). La importancia de cultivar la ética profesional. Recuperado 28 de julio de 2015, de http://www. educamericas.com/articulos/reportajes/la-importanciade-cultivar-la-etica-profesional.
Maldonado, O. (2001). Interdisciplinariedad en bioética. Memorias cátedra Manuel Encizar ética y bioética. Universidad Nacional.

Morin, E. (1996). La transdisciplinariedad, Manifiesto. México: Du Rocher.

Morin, E. (2011). Introducción al pensamiento complejo. España: Gedisa.

Palazzi, C. \& Román, B. (2015). Ética aplicada, entre la recreación moral y la tradición. ARS BREVIS, 11, 176. Recuperado 4 de mayo de 2015, de http://www.raco.cat/index.php/ ArsBrevis/article/view/87656/142429.

Pérez, F. (2008). Ética en el ciberespacio. Ontology Studies, 8, 23-32. Madrid: Universidad de Alicante.

Pérez, R. (2010). El comportamiento moral en las organizaciones: una perspectiva desde la ética de la empresa. Universidad Complutense de Madrid.

Ponce, F. (2011). La modernidad como objeto de indagación filosófica en Jürgen Habermas. A Parte Rei. Revista de Filosofía, 1.

Real Academia Española (s.f.). El concepto de ética. Recuperado de http://lema.rae.es/drae/?val=etica.

Rodríguez, M. (2006). Ética gerencial: Comportamientos éticos de los gerentes que más valoran los empleados en Colombia. Revista Universidad Eafit, 42(143), 45-46.

Tapia, C. (2013). Aspectos éticos en el ejercicio de la profesión. Universidad Latinoamericana de Ciencia y Tecnología. Recuperado de http://bb9.ulacit.ac.cr/tesinas/ Publicaciones/045164.pdf

Valencia, A. (2015). Interdisciplinariedad en ingeniería. Recuperado de http://ingenieria.udea.edu.co/ producciones/ingenieria_sociedad/interdisciplinariedad_ ingenieria.pdf. 